\title{
Les Recettes pour l'enluminure. Do Livro judaico- português de como se fazem as cores
}

Inês Villela-Petit

\section{OpenEdition}

\section{Journals}

Édition électronique

URL : http://journals.openedition.org/medievalista/669

DOI : $10.4000 /$ medievalista.669

ISSN : 1646-740X

\section{Éditeur}

Instituto de Estudos Medievais - FCSH-UNL

Référence électronique

Inês Villela-Petit, "Les Recettes pour l'enluminure. Do Livro judaico-português de como se fazem as cores », Medievalista [En ligne], 9 | 2011, mis en ligne le 02 janvier 2011, consulté le 01 mai 2019. URL http://journals.openedition.org/medievalista/669; DOI : 10.4000/medievalista.669

(C) IEM 
Titulo: Les Recettes pour l'enluminure. Do Livro judaico-português de como se fazem as cores

Autor(es): Inês Villela-Petit

Enquadramento Institucional: CNRS - Centre national de la recherche scientifique - Paris

Contacto: inesvp2000@yahoo.fr

Fonte: Medievalista [Em linha]. №9, (Dezembro 2010). Direc. José Mattoso. Lisboa: IEM. Disponível em: http://www2.fcsh.unl.pt/iem/medievalista/

ISSN: 1646-740X

\section{Resumo}

Guardado na secção hebraica da Biblioteca Palatina de Parma, em Itália, o Livro de como se fazem as cores, é um livro de receitas português para a pintura de livros. Antes de focar o estudo nos seus conteúdos técnicos, vale a pena atentar ao seu contexto literário, que congrega diferentes tradições: ocidental, hebraica, ou mesmo árabe. Este estudo oferece também uma data provável para a obra e sugere ligações a outros manuscritos iluminados na Península Ibérica.

Palavras-chave: Iluminura, cores, pigmentos, Península ibérica

\section{Abstract}

Kept in the Hebraic section of the Biblioteca Palatina of Parma (Italy), the Livro de como se fazem as cores is a Portuguese recipe book for book painting. Before turning to its technical contents, it is worth putting it back in its literary context, at the crossroads of different traditions: Western, Hebrew, or even Arab. The study also offers a likely dating for the book and suggests links with illuminated manuscripts in the Iberian Peninsula.

Keywords: Illuminated manuscripts, colors, pigments, Iberian Peninsula 


\title{
Les Recettes pour l'enluminure. Do Livro judaico-português de como se fazem as cores
}

\author{
Inês Villela-Petit
}

L'œuvre dont il sera ici question ne fait pas partie des collections du Portugal, elle n'est pas non plus un manuscrit enluminé, mais elle a pourtant tout à voir avec l'enluminure portugaise en général, et l'enluminure judéo-portugaise en particulier.

La Bibliothèque palatine de Parme possède sous la cote ms. parm. 1959 (nº 945 de l'ancienne collection De Rossi), un codex sur papier qui contient, entre autres textes, un traité de langue portugaise, quoique transcrit en caractères hébraïques, relatif à la préparation des pigments et des encres pour l'enluminure. Le Livro de como se fazem as cores - tel est son titre - est d'autant plus digne d'intérêt qu'il semble, tant dans la littérature portugaise que dans la littérature juive médiéval, unique en son genre. Nous verrons comment il s'insère malgré tout dans la tradition des réceptaires occidentaux. D.V. Thompson, l'un des meilleurs connaisseurs de ces textes techniques du MoyenAge, avait en son temps souligné le grand intérêt de celui-cil. Cependant, avant d'en considérer le contenu, il n'est peut-être pas inutile, au regard des incertitudes qui pèsent encore sur l'origine de ce traité, de commencer cette présentation en nous attardant un peu sur l'histoire du manuscrit lui-même.

\section{Questions de datation}

L'orientaliste Gian Bernardo De Rossi (1742-1831), professeur à la faculté de théologie de l'Université de Parme de 1769 à 1821, avait rassemblé une riche bibliothèque de

\footnotetext{
${ }^{1}$ D. V. Thompson, Artificial vermilion in the Middle Ages, dans Technical Studies, t. II, 1933, p. 62-70, à la p. 68, note 27 : “A publication uniting text, transliteration and translation, with still further study of this text and its associations, is greatly to be desired".
}

Medievalista online №9| Janeiro - Junho 2011 @ IEM - Instituto de Estudos Medievais 2 
manuscrits hébraïques dont il donna lui-même le catalogue en trois volumes2 en 1803. Ces quelques 1.432 livres, après avoir été proposés sans succès à la Bibliothèque nationale, alors impériale, à Paris3, furent acquis par Marie-Louise d'Autriche dès 1816 et donnés à la Bibliothèque royale de Parme où ils constituent un fonds hébreu de premier plan. Comme plusieurs autres manuscrits originaires de la péninsule ibérique intégrés à la collection parmesane4, il est très probable que celui qui nous intéresse ici soit parvenu en Italie dès l'extrême fin du XVe siècle à la suite des expulsions des communautés juives d'Espagne en 1492, puis du Portugal en 1497. On sait que le duché de Ferrare, le marquisat de Mantoue et la république de Venise comptèrent parmi leurs terres d'accueil.

En dehors du catalogue De Rossi, les références bibliographiques concernant le manuscrit sont succintes et peu nombreuses. Parmi les textes qui le composent, seul a d'ailleurs retenu l'attention le Livro de como se fazem as cores, dont D.S. Blondheim a donné l'édition en caractères hébraïques5 en 1928. La barrière de l'écriture nous a conduit à préférer la translitération du même Blondheim6 parue en 1930 et reprise par A. Moreira de Sà7. D'emblée, la date du codex De Rossi 945 leur pose question. Bien que celui-ci comporte un colophon établissant qu'il aurait été achevé par Abraham fils de Judah ibn Hayyim (ou Chaïm), à Loulé [Algarve] en 5022, soit l'année 1262 du calendrier chrétien, le XIIIe siècle a semblé à tous une datation trop précoce et on a préféré situer le manuscrit à la fin du XVe, voire au XVIe siècle. De fait, un oubli du chiffre des centaines est probable. De là à déplacer son origine du sud du Portugal à la

\footnotetext{
${ }^{2}$ G. B. De Rossi, Manuscripti codices hebraici ejus bibliothecae accurate ab eodem descripti et illustrati, Parme, 1803, en 3 vol. (mss. n ${ }^{\circ} 1$ à 1377), vol. 3, p. 21-22 et 200 ; cf. aussi P. Perreau, Catalogo dei codici ebraici della Biblioteca di Parma non descritti dal De Rossi, 1878.

3 Nous remercions pour cette information Monsieur Michel Garel, conservateur en chef des manuscrits hébraïques à la Bibliothèque nationale à Paris, qui a retrouvé trace de cette acquisition manquée dans les archives du service.

4 On citera notamment une Bible de Huesca en Aragon de la fin du XIIIe siècle, De Rossi n ${ }^{\circ}$ 196, une autre Bible et une Haggada du début du XIVe siècle, respectivement n ${ }^{\circ} 518$ et 1107.

5 D. S. Blondheim, An Old Portuguese work on manuscript illumination, dans The Jewish quaterly review, (new series) 19, 1928-29, p. 79-135, avec une traduction en anglais.

6 D. S. Blonheim, Livro de como se fazem as côres, dans Todd Memorial volumes, philological studies, vol. 1, New York, 1930, p. 71-83.

7 A. Moreira de Sà, O Livro de como se fazem as côres de Abraao b. Judah ibn Hayyim, dans Revista da Faculdade de Letras, IIIe série, $\mathrm{n}^{\circ}$ 4, Lisbonne, 1960. Voir aussi H. J. Abrahams, A thirteenth-century Portugese work on manuscript illumination ("Libro de como se facem as cores"), dans Ambix, vol. 26, 1979, p. 93-99. Le texte est également cité par C. Garzya Romano, I Colori e le Arti dei Romani e la compilazione pseudo-eracliana, Naples, 1996, p. 94, chap. 13, note 1.
}

Medievalista online № 9| Janeiro - Junho 2011 @ IEM - Instituto de Estudos Medievais 3 
Galice espagnole8, il y a un pas qu'on a sans doute un peu hâtivement voulu franchir sur la foi d'un rapprochement onomastique, peut-être séduisant au premier abord, avec l'enlumineur d'une Bible terminée à La Corogne en 1476 : Joseph ibn Hayyim9, mais d'autant plus sujet à caution que ce nom de famille est répandu. Notons aussi à l'appui des indications portées au colophon que sans être une ville de tout premier plan, Loulè, de même que le port de Faro voisin, était dès l'époque musulmane et en tous cas depuis la fin de la reconquête de l'Algarve en 1250, un centre de commerce important, en pleine expansion, semble-t-il, au XVe siècle, avec sa population de mouros forros et surtout son quartier juif (judearia), le Vale judeu de Loulè10.

Dans leurs analyses respectives, nos auteurs n'ont toutefois que peu ou pas tenu compte des ressources de la paléographie et de la codicologie pour amender la date controversée. Cependant, la paléographie hébraïque n'étant pas notre domaine et n'ayant pu de ce fait mener une étude de visu du manuscrit, nous nous contenterons de dire de son écriture qu'elle est une semi-cursive ibérique quelque peu irrégulière et pas des plus soignées, qu'on en rencontre des exemples à partir du XIVe siècle, mais sans qu'une datation plus tardive soit exclue. On peut d'ailleurs en rapprocher un manuscrit hébreu copié à Torres Vedras en 1398 qui comporte dans ses derniers feuillets un acte de vente en portugais et caractères hébraïques, daté de 1446, d'une écriture semi cursive assez proche11. Quant aux filigranes du papier, autres indices à considérer pour établir une datation plausible, ils se révèlent finalement plus décisifs. D'après les renseignements fournis par Madame Nice Ugolotti, de la Bibliothèque palatine de Parme, il sont au nombre de quatre : une couronne (p. 23), une paire de ciseaux (p. 52 et 175), une croix (p. 73) et un filigrane en forme de cornet (p. 44). Si ce dernier motif n'a pu être identifié, la paire de ciseaux est un filigrane exclusivement italien, supposé provenir d'un battoir

\footnotetext{
${ }^{8}$ Ainsi Blondheim : "There is some reason to believe that the present form of the text dates from a period later than the thirteenth century, and that our ms. may have been written in Galicia”, op. cit. p. 98.

9 Bible Kennicott (Oxford, Bodleian Lib., ms. Kennicott 1) terminée par le copiste Moïse ben Jacob ibn Zabara, à Corunna (Galice) en 1482. Celle-ci aurait été faite sur le modèle direct de la Bible de Cervera (1299-1300) enluminée par Joseph Aserfati, aujourd'hui à Lisbonne, Bibl. nac., Il. 72.

10 Voir aussi : M. Kayserling, Geschichte der Juden in Spanien und Portugal, Berlin, 2 vol. 1861 et 1867, II : Die Juden in Portugal ; J. Mendes dos Remedios, Os Judeus em Portugal, Coïmbra, 1895 ; et M. Kriegel, Les Juifs à la fin du Moyen-Age dans l'Europe méditerranéenne, Paris, 1979.

11 Paris, Bibl. nat., ms. hébr. 215, de la main de Joseph b. Guedalia Franco. L'acte au fol. 273 v est aussi de sa main : il établit la vente du manuscrit à un Benveniste habitant de Lisbonne, le 25 décembre 1446. Ce manuscrit nous a été signalé par Monsieur Michel Garel. Cf. C. Sirat et M. Beit-Arié, Manuscrits médiévaux en caractères hébraïques portant des indications de date jusqu'à 1540, Paris-Jérusalem, 1972. Mais voir aussi le ms. hébr. 689, du XIVe siècle.
} 
génois. Comme le second type de ciseaux présenté par Briquet12 (nº 3736 à 3755), elle est dessinée d'un trait simple, sans toutefois que son catalogue donne d'équivalent exact de notre modèle, non plus d'ailleurs que du filigrane en forme de croix pommée posée sur un arc de cercle qui évoque la partie supérieure d'un globe13. La couronne, en revanche, est très proche des $n^{\circ} 4636$ à 4648 de Briquet14, c'est-à-dire, suivant la classification de ce dernier, d'une couronne de type 1 à fleuron central cruciforme et deux demi-fleurons. Le fleuron central, dans les exemples cités en référence, adopte un dessin dit "en fer de lance" dont le modèle se trouve largement répandu en Europe entre 1423 et 1488 . Les deux exemples les plus proches du filigrane du manuscrit parmesan $\left(\mathrm{n}^{\circ} 4645\right.$ et 4646) datent respectivement de 1459-69 et de 1473. Toutes ces variantes de papier à la couronne proviendraient d'un important battoir piémontais. On notera toutefois que, pour établir son catalogue, Briquet n'avait pas rendu visite aux archives d'Espagne et du Portugal, et que par conséquent il semble moins bien renseigné sur la production papetière locale. On dit cependant les papiers espagnols du XIVe siècle dépourvus de filigrane, et dès les années 1330 ils subissent une forte concurrence de ceux, de meilleure qualité, provenant d'Italie, qui devaient les supplanter tout à fait à la fin du siècle15. Bien que d'autres exemples nous manquent d'une diffusion de ce papier à la couronne piémontais dans la péninsule ibérique16, ces quelques considérations étayent l'hypothèse d'une datation basse du manuscrit au XVe siècle, en accord avec

12 C. M. Briquet, Les filigranes, dictionnaire historique des marques du papier dès leur apparition vers 1282 jusqu'en 1600, rééd. Amsterdam, 1968.

13 Des globes surmontés d'une croix figurent dans le catalogue de Briquet : $\mathrm{n}^{\circ} 3031$ et 3032 , et dans $\mathrm{O}$. Valls i Subirà, La Historia del papel en Espana, II, p. 191, fig. 58 (exemple provenant de Càceres, monastère de Nuestra Senora de Guadalupe, XVIe siècle).

14 Des exemples comparables dans : G. Piccard, Die Wasserzeichenkaetei Piccard im Haupstaatsarchiv Stuttgart, vol. I : Die Kronen-Wasserzeichen, Stuttgart, 1970, les n 301 à 327 trouvés en Allemagne (datés entre 1437 et 1470) ; dans Monumenta Chartee papyracece, vol. I : E. Heawood, Watermarks, réimpr. 1950, n 986, 987 et 991 trouvés en Angleterre ; et vol. , Likhachev's Watermarks, réimpr. 1994, $\mathrm{n}^{\circ} 545$ à 1111 (datés 1431 à 1466). En revanche, pas de filigrane de ce type chez M. Zerdoun BatYehouda, Les Papiers filigranés des manuscrits hébreux datés jusqu'à 1450 conservés en France et en Israël, (Brepols, Bibliologia, n 16 : Les Papiers et n 17 : Les Filigranes), 1996.

15 Cf. J. Irigoin, L'Introduction du papier italien en Espagne, dans Papiergeschichte, t. X, Mayence, 1960, p. 29-32. Les registres de la chancellerie d'Aragon témoignent de l'utilisation simultanée des deux types de papier.

${ }^{16}$ La localisation des plus anciens filigranes de la série dans le sud de la France (Lautrec, 1423, Toulouse, 1425 , et Montpellier, 1427) peut en appuyer la présomption, mais nous n'en avons pas rencontré de semblables dans O. Valls i Subirà, La Historia del papel en Espana, 3 vol., en particulier I, Siglos X$X I V, 1978$, et II, Siglos XV - XVI, 1980, aux p. 127-128 et 196 (la couronne $n^{\circ} 75$ de son catalogue de filigranes, probablement aussi d'origine italienne, n'appartient pas au même groupe). Rien non plus dans O. Valls I Subira, Paper and Watermarks in Catalonia, (Monumenta charte, vol. XII), Amsterdam, 1970, 2 vol. La question semble mal étudiée pour le Portugal.

Medievalista online № 9| Janeiro - Junho 2011 ๑ IEM - Instituto de Estudos Medievais 5 
l'écriture et le papier d'importation italienne, soit en corrigeant le colophon $17: 1462$.

\section{Le contexte hébraïque}

Les termes d'une datation post quem offerts par le contenu du manuscrit ne pouvaient être mis à profit puisqu'en dehors de cet Abraham aben Hayyim, par ailleurs inconnu, il s'agit d'extraits d'auteurs des XIIe et XIIIe siècles. Mais le choix des textes qui accompagnent le Livro de como se fazem as cores n'est pas indifférent pour en comprendre les tenants et les aboutissants : ils définissent un contexte intellectuel.

A la suite du traité portugais qui en occupe les vingt premiers feuillets, le manuscrit de Parme comprend dix autres textes distincts18. Parmi ceux-ci figurent des passages de l'œuvre de Moïse Maïmonides (1153 - 1204) dont le chapitre VI de son traité Sefer haMadda (textes $\mathrm{n}^{\circ} 7$ et 8 du manuscrit), des extraits du coutumier juif (minhagim) d'Abraham ben Nathan ha-Yarhi (1155 - 1215), talmudiste provençal originaire de Lunel qui s'établit à Tolède au début du XIIIe siècle (les textes $\mathrm{n}^{\circ} 4,5$ et 6 ), et un emprunt au traité philologique (Mikhlol) de David Kimhi (vers 1160 - 1253), grammairien et exégète de Narbonne (le $\left.n^{\circ} 11\right)$. On y trouve encore trois textes anonymes, et un autre traité d'exégèse juive (masorah) placé, comme le colophon, sous le nom d'Abraham fils de Judah aben Hayyim. Celui-ci semble donc bien être à la fois le copiste du manuscrit, le compilateur de ces textes et, pour certains d'entre eux, l'auteur. Il ne fait vraisemblablement qu'un avec celui qui signe ainsi à la fin du Livro de como se fazem as cores : “Abraham, fils de R. Judah, pur ibérique (sépharade)”.Dans l'ensemble, les textes normatifs et les commentaires grammaticaux retenus par le

17 On ne peut cependant écarter tout-à-fait l'hypothèse d'une copie du XVe siècle, fidèle jusque dans le colophon d'origine, d'un manuscrit plus ancien. La pratique en est attestée, mais la date de 1262 est de toutes façons trop précoce pour le contenu technique du Livro de como se fazem as côres.

18 De Rossi en donne la liste comme suit :

$1^{\circ}$ R. Abr. fil. Jeh. Aben Chaiim 'Liber de modo conficiendorum colorum ad miniandos libros', hispanice [sic].

$2^{\circ}$ Anonymi 'Liber ahibbùr' seu 'intercalationis' cum nonnullis 'excerptis'.

$3^{\circ}$ Anon. 'Ilcoth', seu 'Constitut. libri legis', et 'mesusà' .

$4^{\circ}$ R. Abr. Aben Jarchi 'Constitutiones tesilin' et 'meghillà' ex ej. libro 'Manhigh'.

$5^{\circ}$ Ejusdem 'Constitutiones de repudio'.

$6^{\circ} \mathrm{Ej}$. 'Constitutiones sehudà' seu 'convivii' ex eodem libro 'Manhigh'.

$7^{\circ}$ R. Mosis Maimonidis caput VI libri 'Madàh'.

$8^{\circ} \mathrm{Ej}$. 'Constitutiones legis, tesilin', et 'mesusà'.

$9^{\circ} \mathrm{R}$. Abr. fil. Jeh. Aben Chahiim Liber masoreticus.

'De litteris coronulis ornatis in sacro Pentat. volumine, ac litteris pe convolutis'.

$10^{\circ}$ 'Bakascoth' et 'Pizmonim', seu 'metricae preces'.

$11^{\circ}$ R. David Kimchi 'Excerptum' ex ejus ‘Michlol de nomine’, chart. rabb. 12 a. 1262.

Medievalista online № 9| Janeiro - Junho 2011 @ IEM - Instituto de Estudos Medievais 6 
compilateur relèvent de la massorétique, ce corps de traditions qui définit la façon licite d'épeler, d'écrire et de lire la Bible hébraïque. Lorsque le texte est sacré, son écriture, y compris dans ses aspects les plus matériels que sont l'encre et le support, revêt un caractère religieux 19. Les prescriptions sont donc particulièrement strictes pour la copie du Pentateuque (Torah) et celle d'autres rouleaux de parchemin rituels : livre d'Esther, versets du Deutéronome placés aux linteaux de portes (mezouzot) auxquels sont consacrés les textes $n^{\circ} 3$ et 8 du recueil, phylactères servant à la prière du matin (tephilin), textes $\mathrm{n}^{\circ} 4$ et 8 , et même l'acte de divorce (guet) remis à l'épouse délaissée, texte $\mathrm{n}^{\circ} 5$. Les particularités de la copie du Pentateuque font notamment l'objet du second traité d'Abraham aben Hayyim, son livre massorétique ( $\left.\mathrm{n}^{\circ} 9\right)$ qui, d'après le catalogue de De Rossi20, est tout spécialement consacré aux "litteris coronulis ornatis", ces lettres ornées de signes (tagin) évoquant de petites couronnes.

Nous pouvons nous demander quels rapports entretient le Livro de como se fazem as cores avec ces prescriptions. Alors que l'encre rituelle est une encre noire bien particulière, il n'offre que des encres de couleur. Or, celles-ci, de même que la chrysographie, sont explicitement proscrites pour la copie du Pentateuque. Au contraire des traités massorétiques auxquels il est associé dans le manuscrit parmesan, le réceptaire portugais ne peut donc être destiné à la copie et à l'ornementation des rouleaux synagogaux, mais seulement à l'usage des Bibles et rituels privés, de leurs gloses marginales et de tous les textes profanes. Dans ce contexte, son statut particulier est manifeste. La raison première en est qu'il ne procède pas de la tradition juive, mais puise aux sources de la littérature technique occidentale.

\section{La tradition des réceptaires 21}

Les plus anciens exemples de recueils de recettes techniques occidentaux, écrits en langue grecque sur papyrus, remontent au IVe siècle22. Du VIIIe siècle datent les

\footnotetext{
19 Sur la question des encres rituelles, cf. M. Zerdoun Bat-Yehouda, Les Encres noires au Moyen Age, Paris, 1983, p. 97-121 : "Le problème des encres à usage liturgique chez les Juifs".

20 G. B. De Rossi, op. cit., p. 22 : "De litteris coronulis ornatis in sacro Pentat. volumine, ac litteris pe convolutis".

21 Nous reprenons ici certaines questions que nous avions développées en mars 1999 au cours d'une communication intitulée Sources manuscrites pour l'étude des techniques de la peinture ancienne : les recueils de recettes médiévaux, à l'intention des étudiants-restaurateurs de la Maîtrise de Sciences et Techniques de l'Université de Paris I (conférences Icosaèdre).

22 R. Halleux, Les Alchimistes Grecs, vol. I : Papyrus de Leyde, Papyrus de Stockolm, Fragments de recettes, Paris, 1981.
} 
premiers réceptaires latins qui nous soient conservés : les Compositiones ad tingenda musiva, dites Manuscrit de Lucques23, la Mappae Clavicula dont on trouve mention à cette époque24 et, vraisemblablement, les deux premiers livres du De Coloribus et Artibus Romanorum d'Eraclius25. Il s'agit de compilations hétérogènes de recettes relatives à la métallurgie, la verrerie, la teinture, la peinture, dont la matière nourrit les traités ultérieurs. Certains procédés que l'on retrouvera dans le livre d'Abraham aben Hayyim y sont déjà décrits. On discerne une évolution, à partir du XIIe siècle, vers une plus grande spécialisation de ces recueils techniques et un ordonnancement plus rigoureux de leur contenu comme dans le fameux De diversis artibus de Théophile. Ainsi voient le jour divers traités dévolus au seul domaine de la peinture et couvrant les champs de la peinture sur parchemin et sur panneaux de bois, et de la peinture murale26. Mais ce sont surtout ceux spécifiquement consacrés à l'art de l'enluminure qui doivent ici retenir notre attention. Contemporain de Théophile, le premier traité d'enluminure indépendant est un texte latin de la fin du XIe ou du début du XIIe siècle, intitulé De Clarea et consigné dans un manuscrit malheureusement incomplet de la bibliothèque suisse de Berne27. Il traite des différents modes de préparation du liant au blanc d'oeuf (clarea, on dirait en portugais : clara do ovo) utilisé pour délayer les pigments. Sa seconde partie, consacrée semble-t-il aux mélanges de couleurs, est perdue. Le De Arte illuminandi d'un manuscrit napolitain de la seconde moitié du XIVe siècle est en revanche très complet avec ses quelques 31 chapitres. On y trouve notamment des recettes d'or mussif, de vert d'iris, de rosetta, et de violet de tournesol, tous pigments qui figurent aussi dans le Livro de como se fazem as côres. Ces réceptaires rédigés par et pour des enlumineurs vont se multiplier à la fin du XIVe et au XVe siècle. Dans leur forme comme dans leur contenu, ils s'apparentent aussi au Livro portugais, et notamment à travers l'emploi de la langue vernaculaire : l'allemand pour le

\footnotetext{
23 H. Hedfors, éd., Compositiones ad tingenda musiva, Uppsala, 1932.

24 J.G. Hawthorne et C.S. Smith, The Mappae Clavicula : a little key to the world of mediaeval techniques, dans Transactions of the American Philosophical Society, new series 64, 1974. Comme celles des Compositiones, les recettes de la Mappae Clavicula sont translitérées de textes grecs plus anciens.

25 C. Garzya Romano, Eraclio, i colori e le arti dei Romani, Naples, 1996.

26 Outre le livre I de Théophile, citons le De Coloribus et mixtionibus (XIIe siècle), le De Coloribus naturalia exscripta et collecta (fin du XIIIe-début XIVe siècle), ou encore le De Coloribus faciendis de Pierre de Saint-Omer et le Liber de coloribus illuminatorum sive pictorum (XIVe siècle), et le plus célèbre d'entre eux : le Libro dell'arte de Cennino Cennini.

27 Cf. R.E. Strauss, De Traktat De Clarea in der Burgerbibliothek Bern : eine Anleitung für Buchmalerei aus dem Hochmittelalter, dans Jahresbericht des Schweizerisches Institut für Kunstwissenschaft, 1964, p. 89-114.
}

\section{Medievalísta online №9| Janeiro - Junho 2011 @ IEM - Instituto de Estudos Medievais 8}


manuscrit de Göttingen, écrit à Mayence dans le second quart du XVe siècle28, ou l'italien du Ricettario per pittura su libro rédigé entre 1433 et 1437 par le moine chartreux Bartolomeo da Siena29, et des Ricepte daffare piu colori de 1462 d'un autre enlumineur siennois, Ambrogio di ser Pietro30. On voit par là la diffusion de cette littérature à l'horizon des ateliers européens. Ainsi le traité judéo-portugais participe-t-il de ce double mouvement d'une spécialisation des réceptaires et de l'adoption des parlers locaux.

S'il trouve pleinement sa place dans cette tradition de traités technico-artistiques que nous venons d'évoquer brièvement, le Livro de como se fazem as cores est cependant, on l'aura compris, original à plus d'un titre. Il l'est d'abord par sa langue, puisque c'est à ce jour le seul réceptaire médiéval en langue portugaise connu, et il l'est aussi par sa culture d'origine, la culture juive. Celle-ci ne se manifeste pourtant nullement dans le contenu du traité, mais seulement par les particularités externes de sa graphie et du contexte du manuscrit parmesan. C'est par là, et non par son contenu, qu'il se distingue des autres traités pour l'enluminure et la peinture que nous avons cités qui, quant à eux, appartiennent peu ou prou, et parfois très explicitement, au domaine de l'art chrétien, tant monastique que laïque. Qu'on pense à Théophile, pseudonyme du moine bénédictin Roger de Helmarshausen. Notre traité puise sa matière à ces mêmes sources, son contenu technique et le formulaire de ses recettes se calquent sur cette tradition, mais en lui faisant place dans son recueil de commentaires exégétiques, Abraham aben Hayyim, qui fait ici figure autant de lettré que de copiste ou d'enlumineur, l'a intégrée et assimilée aux pratiques du livre et de l'enluminure hébraïque.

\section{Une influence arabe?}

Du Livro de como se fazem as cores, nous n'aborderons que brièvement l'aspect

28 H. Lehmann-Haupt, The Göttingen Model Book, a facsimile edition and translation of a fifteenth century illuminator's manual, Columbia, 1972. Pour la peinture sur panneaux, il faut aussi citer le manuscrit de Strasbourg (allemand, première moitié du XVe siècle).

29 B.S. Tosatti Soldano, Miniature e vetrate senesi del secolo XIII, dans Collana storica di fonti e studi diretta da G. Pistarino, 25, Gênes, 1978, p. 139-149.

30 D.V. Thompson, The Ricepte daffare piu colori of Ambruogio di Ser Pietro da Siena, dans Archéion, XV, 1933, p. 339-347. En italien aussi : le célèbre Libro dell'arte de Cennino Cennini (fin du XIVe siècle), et les Segreti per colori (milieu du XVe siècle). On trouve également des recettes en français dans le Liber colorum, dit recueil de Jean Lebègue (1431), cf. I. Villela-Petit, La Peinture médiévale vers 1400, autour d'un manuscrit de Jean Lebègue (édition du Liber colorum), thèse pour le diplôme d'archiviste paléographe, 1995.

Medievalista online №9| Janeiro - Junho 2011 ๑ IEM - Instituto de Estudos Medievais 9 
proprement linguistique et lexical. Il requiert l'examen d'un spécialiste de philologie lusitane que nous ne sommes pas. Le vocabulaire technique, noms d'ingrédients et d'ustensiles, est largement emprunté à l'arabe. Nous avons relevé près d'une trentaine de mots de cette provenance31, alors déjà assimilés par la langue portugaise et dont beaucoup sont passés au portugais contemporain comme azinhavre, le vert-de-gris, alvaiade, le blanc de plomb, ou zarcão, le minium de plomb. D'autres emprunts sont faits au français (arjen, branchete pour "blanchet" ancien nom du blanc de plomb), ainsi qu'au vocabulaire alchimique : jupiter - l'étain, fugitive - le mercure, lune - l'argent, qui proviennent sans doute des sources utilisées pour la compilation. On obtient ainsi des doublets tels que jupiter / estanho, alvaiade / branchete, voire trois termes pour désigner une même substance : fugitivo / arjen vivo / azougue, mais toujours le terme arabe sert à expliciter l'autre, ainsi fugitivo id es[t] azougue. Le réceptaire portugais est donc au confluent d'influences très diverses.

La présence de termes arabes dans le texte et peut-être aussi les chapitres consacrés au jeu d'échecs (21) ou au décor des lames (16), invitent d'ailleurs à considérer une autre source possible à la matière du traité : la littérature technique arabe. Si les textes arabes consacrés à la pratique artisanale, à l'inverse de l'abondante littérature alchimique, sont peu nombreux, il en est pourtant un particulièrement riche pour notre propos puisqu'il envisage la fabrication du livre dans ses différents aspects, de la préparation des plumes pour l'écriture aux techniques de la reliure. Cette somme en douze chapitres, Le Soutien des scribes et les outils du savoir, a été composée vers 1025 par Ibn Badis (1007 1061), prince de la dynastie royale des Zirides de Kairouan. Avec le développement de l'art de la calligraphie, les Arabes ont nourri pour l'encre une passion certaine qui rend Ibn Badis particulièrement prolixe sur le sujet de leur composition : encres noires, encres de couleur, encres métalliques et sympathiques. Son traité, en particulier les chapitres IV consacré aux encres de couleur et VI aux teintures et aux mélanges de pigments, constitue de ce fait un terme de comparaison intéressant avec le Livro de como se fazem as cores où nous devons noter la place faite aux encres colorées. Ainsi, dans le Soutien des scribes, une encre "comme l'or rouge", mêlant au safran le réalgar (un sulfure d'arsenic), et une autre y ajoutant de l'orpiment (autre sulfure d'arsenic), ne

31 Mots d'origine arabe relevés dans le texte : açafrao, açumbres, alacar, alcrevite, alfadida, alfer, algodâo, alguidar, almartaque, almofariz, altamia / atalmia, alvagen, alvaialde, anoxatar, axedres, azarcâo, azarnefe, azeite, azinhavre, azogue, azul, carmin, çufii, malega (?), marfil. 
sont pas sans rapport avec la recette 31 pour une encre à base de safran et d'orpiment 32 . Mais, outre la plus grande variété des produits végétaux qui entrent dans leur composition (grenade, myrte, mirobolan, coriandre, anémones, basilic..), les encres colorées d'Ibn Badis ont une parenté étroite avec ses encres noires de type métallogallique par l'emploi presque systématique qu'elles font de la noix de galle (ce sont donc des encres tanniques), alors que les encres de couleur du Livro de como se fazem as cores ne mentionnent pas celle-ci et ne diffèrent donc pas dans leur composition des pigments pour la peinture. Plusieurs de ces recettes peuvent d'ailleurs être employées indifféremment comme encre ou comme peinture. De plus leur formulaire relève pleinement de la tradition occidentale, alors que la structure des recettes arabes est assez différente, ce qui exclut toute filiation directe. Si influence arabe il y a, elle est plus diffuse et transparaît peut-être dans une certaine propension de l'auteur du Livro de como se fazem as cores à caractériser les couleurs par des métaphores telles que azul como cardeo (6 - bleu comme le chardon), color preto e fermoso que semelhe azevinhe (19 - beau noir comme le jais33), bleu celeçtre (29), cor de nuvem (33 - couleur de nuage) et, plus courant, color sanguinea (30), propension poétique qui était particulièrement marquée chez Ibn Badis : encres bleu de paon, encres couleur de rubis, couleur du ciel, couleur sang de gazelle, couleur des dates mûres, des jeunes fauves, des fleurs de grenade... Mais il est temps maintenant d'examiner plus avant le contenu du réceptaire portugais.

\section{Contenu du traité}

Le Livro de como se fazem as cores est donc un recueil de 45 recettes pratiques pour la fabrication des pigments et des encres, para aluminar os livros nous dit le prologue : Aqui se comiença o livro de como se fazem as cores, das tintas todas pera aluminar os livros.... Des lacunes dans le manuscrit nous privent du texte de deux de ces recettes 34 . Sur les 43 restantes, six introduisent un élément d'hétérogénéité dans le propos puisqu'elles ne concernent pas l'enluminure mais la décoration des lames d'épées, la teinture de leurs manches de bois ou d'os et la réalisation des pièces d'ivoire du jeu

\footnotetext{
32 L'origine du procédé est ancienne puisqu'on trouve déjà dans le papyrus de Leyde une encre à base d'orpiment doré, safran, poudre de cristal de roche et blanc d'œuf ou gomme, cf. R. Halleux, op. cit., $\mathrm{n}^{\circ}$ 56. De même, dans la Mappae Clavicula, le chapitre 45.

33 Il s'agit plutôt ici d'azeviche, le jais, que d'azevinho, le houx.

34 Les numéros 22 et 23. Sont aussi manquants la fin de la recette 21, le début de 24 et un passage de 26.
} 
d'échecs (16 à 21). Autour de la césure que constituent ces dernières, on peut répartir les recettes en deux parties qui correspondent peut-être à deux temps dans la composition du réceptaire. La première (recettes 1 à 15) porte uniquement sur la confection des pigments. Ceux-ci sont regroupés par couleurs : en premier lieu l'or et ses substituts (1 à 4), puis le bleu (5 à 7), le rose et l'orangé (8 à 10), le vert (11 et 12), et le rouge (13 à 15), selon un ordre que l'on retrouve grosso modo dans d'autres recueils35. La seconde partie (recettes 24 à 45) est moins ordonnée et juxtapose de nouvelles recettes, des indications sur les liants, la colle, le vernis, des mélanges de pigments et des indications d'usage. Il est possible qu'elle procède d'une source distincte car les premiers mots de la recette 25 ressemblent à un prologue de réceptaire : Quien quixer obrar con oro e con otras cores e como se deven a fazer e a miçerar...

En ajoutant aux recettes proprement dites les pigments dont il est seulement fait mention, on obtient une palette étoffée : feuille d'or avec diverses assiettes ou sisa (3, $25,36,38,39$ et 43)36, or par attrition (4), argent (39 et 42), étain doré ou vernis (3 et 42 ), et or dit mussif ou mosaïque, oro musico, qui est un composé de soufre et d'étain (1 et 2), lapis lazuli (7), bleu d'argent (5 et 6), indigo (33), et violet de tournesol (24), rouge vermillon composé de soufre et de mercure (15), rouge de plomb (10), différentes laques roses de bois de brésil $(8,9,27,44)$ et des laques de kermès ou de cochenille, carmin, $(13,14,30)$, des verts de cuivre $(11,12,28)$ et un vert d'iris $(45)$, des jaunes d'ocre, de safran (31) et d'orpiment (32), enfin le blanc de céruse et le noir de noyaux de pêche. Cette palette est plus large que la liste des dix couleurs fournie au chapitre 27 dans laquelle il faut peut-être voir les plus utilisées : Sabe que dez são as côres principais : azul, oripimento e vermelhon, verde, carmin çufii37, catasol, açafrão, azarcon, alvaialde, brasil... (bleu, orpiment et vermillon, vert, carmin, tournesol, safran, minium, blanc de plomb, brésil). Elle nous semble en tous cas assez caractéristique de la

\footnotetext{
35 Voir par exemple celui des Scripta colorum, cf. F. Tolaini, Incipit Scripta Colorum : un trattato contenuto nel ms. 1075 della Biblioteca Statale di Lucca, dans Critica d'Arte, LVIII, n 3 et 4, 1995, p. 54-68 et 47-56.

36 Dans la préparation de l'assiette pour la feuille d'or, l'adjonction de poudre de noyaux de pêches brûlés est assez originale. On retiendra aussi le fait que la lessive employée soit toujours une lessive de vigne. Mais ces indices qui feraient "couleur locale" sont limités.

37 Le mot arabe 'çufi' signifie laine ou flocon de laine (nos remerciements vont à Mademoiselle Hélène Vuidel, arabisante, pour son aide dans l'élucidation de ce mot). Contrairement aux éditeurs du Livro qui en font un onzième nom de couleur, il faut lier ce terme à celui qui précède : 'carmin çufii' sans doute au sens de bourre de draps de laine teints au kermès que les peintres récupéraient pour leur usage et dont ils extrayaient le colorant. C'est la cimatura scarlate des textes latins.
} 
seconde moitié du XIVe siècle et du début du XVe siècle, période au cours de laquelle fait aussi son apparition dans les réceptaires un pigment comme l'or mussif38, tandis que le lapis lazuli et les laques de brésil39 sont particulièrement en faveur. Rappelons incidemment que, sous le nom de brésil (brasilium), des bois rouges tinctoriaux du type bois de sappan (Cæsalpinia sappan L.) étaient importés en Europe des bords de l'Océan indien depuis le XIIe siècle, bien avant que Pedro Alvares Cabral en découvrit de semblables essences sur les côtes d'un pays qui allait devenir le Brésil. Représentatifs aussi de la palette médiévale sont les colorants emmagasinés sur des morceaux de tissus, tels le jus d'iris et les extraits de Crozophora tinctoria L., plante méditerranéenne appelée vulgairement tournesol (le catasol du texte), et en latin folium. En revanche, on ne trouve pas trace dans le recueil de pigments qui ne commencent à être employés qu'à partir du XVe siècle.

A côté de ces pigments pour aluminar, pintar ou encore perfilar (tracer le contour) qu'il convient de poser au pinceau (con pinzel), 20\% des recettes se réfèrent explicitement à leur utilisation comme encre para escrever. Ce sont des encres dorées à l'or mussif, des encres bleues (bleu d'argent semble-t-il, et lapis-lazuli), rouges de brésil et jaunes de safran, sans compter les lettres faites avec la feuille d'or. Si l'enluminure utilise simultanément la plume et le pinceau fin, le trait coloré et l'à-plat de couleur, il n'est pas interdit de voir aussi dans cette forte proportion des encres de couleur un reflet de la place prépondérante de l'écriture hébraïque sur l'image, avec ce compromis si particulier qu'est la micrographie, image uniquement créée par l'écriture, utilisée souvent pour recopier les commentaires exégétiques, et qui admet la couleur. On en rencontre à l'encre rouge, à l'encre violette... De même, l'enluminure juive partage le goût encore si répandu dans les ateliers portugais des XIVe et XVe siècles pour les lettres ornées de filigranes et les panneaux filigranés à l'encre noire, rouge, violette, bleue ou verte.

Cependant, en plus de cette variété de composés chimiques, de minéraux et de laques

\footnotetext{
38 Dans un manuscrit du XIIIe siècle (Londres, Brit. Lib., ms. Egerton 840 A), une recette incomplète du Pseudo-Eraclius passe pour être une recette d'or mussif, mais il y manque au moins la mention du soufre : Deauratura efficitur de vivo argento et stanno, ita ut tres partes sint de vivo argento et quatuor de stagno. En dehors de celle-ci, l'une des plus anciennes est celle fournie par le De Arte illuminandi (seconde moitié du XIVe siècle).

39 A propos des laques rouges, I. Villela-Petit, Brésil et autres rouges : dix recettes de laque médiévales, dans Technè, n 4, 1996, p. 68-73. [Voir aussi P. Roger, S. Vandroy et I. Villela-Petit, "Les laques de brésil dans l'enluminure médiévale: reconstitution à partir de recettes anciennes", dans Studies in Conservation, t. $48, \mathrm{n}^{\circ} 3,2003$, p. 155-170].
} 
d'origine animale ou végétale, le Livro de como se fazem as cores indique aussi plusieurs mélanges (miçeramentos) : - laque de brésil et bleu (cor india), — laque et noir (pinta negra), — laque de brésil, blanc et noir (indio alvo), — vert de cuivre et safran, - vert et céruse (sonbra verde e alvo), — bleu et céruse (celeçtre ou azul branco), - bleu et carmin (cor sanguinea), — safran et orpiment (cor semelhavel au safran seul), - indigo et blanc (cor de nuven), — bleu, carmin et blanc (cor de azul e carmin), — vermillon et jaune (ocre, ocre colorado), — vermillon, jaune et noir (ocre colorado en negro). Ces mentions de mélanges, qui dans la tradition technique médiévale sont plus rares que les recettes de fabrication proprement dite, rappellent celles d'un réceptaire anonyme du XIVe siècle, le Liber de coloribus illuminatorum sive pictorum, sans que toutefois elles soient superposables40. L'éventail des tons et des nuances est quelque peu différent, mais on note dans la désignation des couleurs résultantes une habitude lexicale commune qui consiste à juxtaposer deux termes premiers, ainsi album viride ou verde e alvo. D'un grand intérêt aussi sont les indications d'usage contenues dans cette seconde partie du Livro portugais aux chapitres 31, 34 et surtout 35 qui leur est exclusivement consacré41. Bien que leur destination ne soit pas explicite ici, il s'agit à l'origine de conventions picturales pour la représentation du vêtement et des drapés. Elles apparaissent dans un traité remontant au XIIe siècle, dit De coloribus et mixtionibus, qui a été très largement recopié jusqu'au XIVe siècle compris42. Il fournit des formules du type : Azurum misce cum albo, incide de puro azuro, matetiza de albo plumbo. Il s'agit en fait de dessiner les plis (incidere) d'un ton de bleu sombre dans la surface bleu clair du vêtement qu'on veut représenter, et d'y ajouter des rehauts de lumière en blanc pur (matizare) afin de donner du relief au drapé. Dans le

40 Cf. I. Villela-Petit, Copies, remaniements et renouvellement dans les réceptaires du Bas Moyen-Age : ... 2. Le Liber de coloribus illuminatorum, un réceptaire anonyme du XIVe siècle, à paraître dans les actes du colloque de 1997 sur Lo Studio dei ricettari tecnico-artistici medievali, Pise (Scuola Normale Superiore) [finalement publié sous le titre "Copies, Reworkings and Renewals in Late Medieval Recipe Books", dans J. Nadolny éd., Medieval Painting in Northern Europe : Techniques, Analysis, Art History, Studies in commemoration of the $70^{\text {th }}$ birthday of Unn Plahter, Londres, 2006, p. 167-181].

${ }^{41}$ Les indications d'usage sont les suivantes : Se quizeres matizar con el [o açafrão] do azul been moido, quanto a terça parte, e não mais (chapitre 31). ...e deita sobre el [o azul] para matiza-lho carmin o do brasil (chapitre 34). Se quizeres colorar con azul branco, matiza con azul puro. E se quizeres colorar con carmin, matiza con carmin o con brasil o con vermelhon. E se quizeres colorar indio alvo, matiza en el con verde puro. E se quizeres colorar con azarcon, matiza sobre el carmin o brasil e vermelhon. E se quizeres colorar con vermelhon, matiza con brasil o con carmin. Pero as cores todas se podem matizar con negro (chapitre 35).

42 Voir Fr. Tolaini, op. cit. ; et I. Villela-Petit, Imiter l'arc-en-ciel : la règle des couleurs dans la Schedula diversarum artium de Théophile, dans Histoire de l'Art, n 39, 1997, p. 23-36. 
Livro de como se fazem as côres, la formule devient par exemple : Se quixeres colorar con azul branco, matiza con azul puro. Cet usage et l'origine du mot matizare ont été précisément étudiés par E. Webster-Bulatkin43. Il faut toutefois insister sur le sens que le portugais matizar prend dans notre traité : il ne correspond pas encore au sens littéraire de "émailler de couleurs variées" qu'on lui connaît dans la poésie espagnole à partir du XVe siècle, mais il a déjà perdu son sens technique initial de "faire des rehauts clairs". Il paraît même signifier à l'inverse, supplantant en ce sens le terme latin incidere, puisque le réceptaire ajoute : Pero as cores todas se podem matizar con negro. En fait, au couple incidere de sombre et matizare de clair, se substitue celui de colorar (peindre une surface) et matizar (qui reprend les sens des deux verbes latins, aussi bien tracer les plis que faire les rehauts). Derrière l'identité lexicale se devinent des conventions de représentation sensiblement différentes : alors que le De coloribus et mixtionibus était fondé sur un mode ternaire (surface en ton moyen, trait foncé, rehaut clair), le Livro de como se fazem as cores semble nous ramener au mode binaire (surface claire, trait sombre).

\section{L'exemple de la Haggadah de Londres}

Il nous reste enfin à esquisser quelques rapprochements possibles entre ces recettes et indications d'usage, et la production de manuscrits enluminés contemporaine. Les recueils de recettes - leur large diffusion et le Livro de como se fazem as cores en témoignent - sont un patrimoine technique commun aux ateliers artistiques tant chrétiens que juifs. De plus, l'influence souvent forte des styles locaux sur l'art juif ne permet pas de le considérer isolément, ni au point de vue stylistique, ni a fortiori au point de vue des pratiques picturales. De ce fait, le réceptaire pourrait être mis à contribution aussi bien pour l'étude de l'enluminure hébraïque que pour celle de l'enluminure portugaise en général, voire ibérique. Mais, comme son contexte culturel nous y invite, c'est d'abord dans les manuscrits hébraïques qu'il convient de chercher des points de convergence.

Nous avons déjà relever l'importance des encres colorées, qui peut être mise en rapport

43 E. Webster Bulatkin, The Spanish word 'matiz', its origin and semantic evolution in the technical vocabulary of medieval painters, dans Traditio, vol. X, 1954, p. 459-527, aux p. 491-492. 
avec la production de Bibles hébraïques lisboètes de la fin du XVe siècle44, aspect le mieux connu de l'enluminure judéo-portugaise. Cependant, le texte du réceptaire laisse toute sa place à l'éventualité de la représentation figurative. Or, comme nous l'avons déjà remarqué, ces recettes présentent un certain conservatisme qui autorise à élargir le champ de la recherche en amont. L'enluminure juive ibérique du XIVe siècle montre plusieurs tendances : à côté de la persistance du décor aniconique et du goût pour la micrographie, parallèlement aussi aux emprunts à l'art chrétien des lettres ornées et des rinceaux peuplés venus de France, elle voit se développer de grands cycles d'enluminures figurées dans des manuscrits à usage familial que sont les Haggadah, rituels pour la Pâque juive45. Les plus célèbres sont la Haggadah de Sarajevo46 (Aragon, après 1350) et la Haggadah d'or47 ([Barcelone], vers 1320), illustrées de nombreuses scènes bibliques et liturgiques. Mais celle dont la palette nous a parue la plus intéressante à mettre en rapport avec le Livro de como se fazem as cores est une haggadah de la British Library, ms. Or. 2737, généralement située dans la première moitié du XIVe siècle en Castille (?)48. Elle comprend un cycle de 66 enluminures pleines pages sur fond de parchemin en réserve. Les scènes sont délimitées par des rinceaux détourés de rouge vif ou de violacé qui se terminent dans toute leur partie haute par des représentations d'architecture vivement colorées juxtaposant leurs tours, dômes, clochetons, gâbles et créneaux en un mélange de réminiscences mozarabes et d'influences gothiques. Tandis que les visages sont simplement dessinés à l'encre, les vêtements et le décor architecturé montrent des tons de rouge, d'orangé, de bleu terne et de vert, en une palette assez large. Ainsi dans la scène des Retrouvailles (fol. 267), les plis des vêtements orangé vif ou violet transparent sont modelés de traits noirs qui rappellent l'indication du Livro de como se fazem as cores que le noir peut matizar sur toute couleur. Dans celle de la Purification des récipients (fol. 90), le bleu-gris délavé

44 Cf. G. Sed-Rajna, Manuscrits hébreux de Lisbonne, un atelier de copistes et d'enlumineurs au XVe siècle, Paris, 1970 ; Th. Metzger, Les manuscrits hébreux copiés et décorés à Lisbonne dans les dernières décennies du XVe siècle, Paris, 1977.

45 A propos des Haggadah enluminées, on consultera : J. Gutmann, The Illuminated medieval Passover Haggadah : investigations and research problems, dans Studies in Bibliography and Booklore, VII, 1965, p. 3-25 ; M. Metzger, Deux fragments d'une haggadah espagnole du XIVe siècle, dans Gesta, VI, 1967, p. 25-34 ; et du même auteur, La Haggada enluminée, Leyde, 1973.

46 Cf. C. Roth, La Haggadah de Sarajevo, 1963.

${ }^{47}$ Londres, Brit. Lib., ms. Add. 27210 ; cf. B. Narkiss, The Golden Haggadah, Londres, 1970.

48 Cf. B. Narkiss, Hebrew illuminated manuscripts in the British Isles, 2. vol. : The Spanish and Portuguese manuscripts, Jérusalem - Londres, 1982, notice $\mathrm{n}^{\circ} 9$ 
utilisé pour représenter l'eau n'est pas sans évoquer la "couleur nuage", cor de nuven faite de blanc et d'une pointe d'indigo. On trouve de même cette eau de bleu-gris, peutêtre d'indigo passé en lavis, dans la représentation du Déluge de la Haggadah de Sarajevo. A défaut d'analyses physico-chimiques, il n'est pas possible de donner une identification formelle des pigments observés, gageons cependant que plusieurs doivent correspondre à la palette d'Abraham aben Hayyim, tels les orangés de minium, les verts de cuivre, les laques violacées, les bleus ternes d'indigo... En comparaison d'autres exemples, dont la Haggadah d'or, qui épouse le style gothique avec une grande maîtrise, celui de cette haggadah de Londres pourrait être qualifié de provincial. Elle ne manque pourtant pas de charme, de fraîcheur et de vivacité dans la peinture de ces scènes rituelles et de la vie quotidienne. L'absence de manuscrits comparables en rend toutefois la datation et la localisation délicate.

Il est devenu courant de placer généreusement en Castille les manuscrits hébraïques non localisés et dont le style ne permet pas de les rattacher à ceux mieux connus de Navarre, de Catalogne ou d'Aragon. Pourtant, il se pourrait que certains d'entre eux proviennent aussi du Portugal, qui eut lui-aussi des communautés prospères et des personnages hautplacés dans l'administration royale susceptibles de faire réaliser des manuscrits hébraïques enluminés, mais dont l'art est très mal connu avant les grandes bibles ornées de la seconde moitié du XVe siècle. L'existence même du réceptaire judéo-portugais inviterait pourtant à reconsidérer certaines de ces attributions. En attendant de voir ressurgir d'autres témoins d'une enluminure juive au Portugal, une étude linguistique et une nouvelle édition de l'œuvre d'Abraham aben Hayyim seraient bienvenues.

Nous tenons spécialement à remercier Monsieur Michel Garel, conservateur en chef des manuscrits hébraïques à la Bibliothèque nationale à Paris - Département des manuscrits orientaux, pour l'aide précieuse et bienveillante qu'il nous a apportée dans l'étude du Livro de como se fazem as côres. 
Les Recettes pour l'enluminure. Do Livro judaico-português de como se fazem as cores - Inês Villela-Petit

\section{Liste des recettes}

\section{do Livro de como se fazem as cores}

\begin{tabular}{|c|c|}
\hline 1. or mussif / oro musico & 24 violet de tournesol / nano do catasol \\
\hline 2. or mussif / oro musico & 25. assiette pour l'or \\
\hline 3. feuille d'or / oro & 26. liant pour le bleu \\
\hline 4. dorure par attrition / oro & 27. rose de brésil et mélanges \\
\hline 5. bleu d'argent / nobre azul & 28. liant pour le vert \\
\hline 6. bleu d'argent / azul & 29. liant pour le bleu \\
\hline 7. bleu de lapis lazuli / azul de Acre & 30. laque de kermès / bom carmin \\
\hline 8. laque rose de brésil / rosa & 31. jaune de safran / açafrao \\
\hline 9. autre laque rose de brésil / otra rosa & 32. jaune d'orpiment / oripimento \\
\hline 10. rouge minium / azarcon & 33. bleu indigo / negro anil \\
\hline 11. vert de cuivre / azinhavre & 34. liant pour le bleu \\
\hline 12. autre vert de cuivre / otro azinhavre & 35. les ombres \\
\hline 13. laque rouge de kermès / nobre carmin & 36. assiette pour la feuille d'or \\
\hline 14. autre laque de kermès / otra carmin & 37. les mélanges /mixeramentos das côres \\
\hline 15. rouge vermillon / vermelhon & 38. assiette de l'or \\
\hline 16. dorer épées et couteaux & 39. pour brunir or et argent \\
\hline 17. teinter os et bois & 40. colle de parchemin \\
\hline 18. teinter l'os en rouge & 41. un orangé / ocre \\
\hline 19. teinter le buis en noir & 42. vernis / barniz \\
\hline 20. teinter l'os en vert & 43. or à la mixtion \\
\hline 21. pièces du jeu d'échecs en ivoire & 44. laque rose de brésil / boa roseta \\
\hline fin effacée, jusqu'à 24 & 45. vert d'iris / bom verde \\
\hline
\end{tabular}




\section{COMO CITAR ESTE ARTIGO}

\section{Referência electrónica:}

VILLELA-PETIT, Inês - “Les Recettes pour l'enluminure do Livro judaico-português De como se fazem as cores”. Medievalista [Em linha]. º9, (Dezembro de 2010). [Consultado dd.mm.aaaa]. Disponível em http://www2.fcsh.unl.pt/iem/medievalista/MEDIEVALISTA9lpetit9005.html. ISSN 1646-740X.

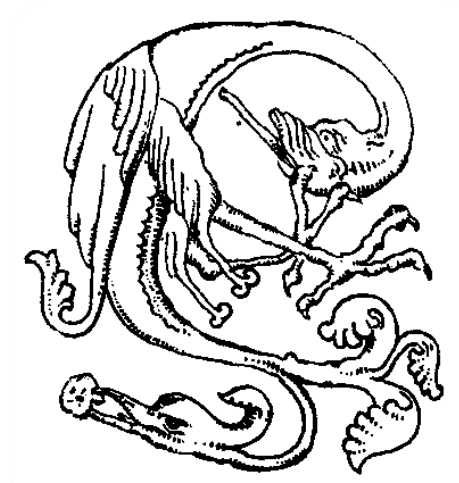

